\title{
A Speedup Method for SVM Decision
}

\author{
Yongsheng Zhu', Junyan Yang ${ }^{1}$, Jian $\mathrm{Ye}^{2}$, and Youyun Zhang ${ }^{1}$ \\ ${ }^{1}$ Key Laboratory of Education Ministry for Modern Design and Rotor-Bearing System, \\ Xi' an Jiaotong University, Xi' an 710049, China \\ \{azhu2005, yjunyan\}@gmail.com, \\ yyzhang1@mail.xjtu.edu.cn \\ ${ }^{2}$ Network \& Information Technology Center of Library, Xi' an Jiaotong University, \\ Xi'an 710049, China \\ yejian@mail.xjtu.edu.cn
}

\begin{abstract}
In this paper, we proposed a method to speed up the test phase of SVM based on Feature Vector Selection method (FVS). In the method, the support vectors (SVs) appeared in the decision function of SVM are replaced with some feature vectors (FVs) which are selected from support vectors by FVS method. Since it is a subset of SVs set, the size of FVs set is normally smaller than that of the SVs set, therefore the decision process of SVM is speeded up. Experiments on 12 datasets of IDA show that the number of SVs can be reduced from $20 \%$ to $99 \%$ with only a slight increase on the error rate of SVM by the proposed method. The trade-off between the generalization ability of obtained SVM and the speedup ability of the proposed method can be easily controlled by one parameter.
\end{abstract}

\section{Introduction}

Support Vector Machines (SVM) is a new type learning machine introduced by V.N. Vapnik and et.al.[1], and was broadly studied and applied in many fields for the comparable classification ability to traditional learning machines and good theoretical bases. However, SVM is slower in test phase than other learning machines such as neural network and decision trees [2-4].

To tackle this problem, several methods were introduced. The decision function of SVM is a weighted linear combination of support vectors (SVs) in feature space[1], therefore the decision speed of SVM is proportional to the number of support vectors and most proposed methods try to accelerate the test phase of SVM by reducing the number of support vectors. The methods can be separated into two types. The first type is pre-processing method, in which some special procedures are adopted before or during training SVM, such as processing the training samples[5], reformulating the training problems of SVM [6,7], as well as adoption of special training strategies during training SVM[8,9]; the second type is post-processing method in which the SVM is firstly trained in the normal way, and some additional process are directly applied to the support vector set after training to reduce the number of SVs. This type method is represented typically by the Reduced Set method [2,4,10], as well as methods reported in [11-13]. Although it can also speeds up the training phase in some degree[ 8,9$]$, pre-processing methods always deal with the entire training sample 
set in most cases; while post-processing methods just operates on the SVs set, so it is easier in implementation and more practical than pre-processing methods. On the other hand, since post-processing methods directly reduces the size of SVs set, it is more "natural".

In this paper, a new post-processing method is proposed to simplify support vector solutions. In the method, the support vectors(SVs) used by the decision function of SVM are replaced by a subset(named feature vectors, FVs) of SVs set which is selected with Feature Vector Selection Method(FVS)[14]. Experimental results on several standard datasets show that the proposed method can speed up SVM from $20 \%$ to $99 \%$ with small loss on generalization ability of SVM, and the trade-off between the speedup ability and the performance can be easily controlled by one parameter.

The rest of the present paper is organized as follows. In the second section, the FVS method is briefly described, the decision function of SVM expressed with FVs is also included in this section. Experiments and discussions are described in section 3 and section 4 separately. In section 5, we conclude the paper.

\section{Feature Vector Selection Method}

\subsection{Feature Vector Selection Method}

Feature Vector Selection Mehtod(FVS) only involves the operation on the kernel matrix[1] which is defined as:

$$
\mathbf{K}=\left(k_{i j}\right)_{1 \leq i, j \leq M}
$$

where $M$ is the number of samples $\mathbf{x}_{i}, i=1, \cdots, M, k_{i j}=\boldsymbol{\Phi}^{t}\left(\mathbf{x}_{i}\right) \boldsymbol{\Phi}\left(\mathbf{x}_{j}\right)$, $\boldsymbol{\Phi}: \mathbf{X} \rightarrow \mathbf{F}, \mathbf{x} \rightarrow \boldsymbol{\Phi}(\mathbf{x})$ is a mapping operation from the input space $\mathbf{X}$ into a feature space $\mathbf{F}$. The aim of FVS is to find a basis of $\boldsymbol{\Phi}\left(\mathbf{x}_{i}\right), i=1, \cdots, M$ so that each sample can be expressed in $\mathbf{F}$ with the basis.

Let $L$ be the number of selected vectors (named feature vectors in [14], FVs), note $\boldsymbol{\Phi}\left(\mathbf{x}_{i}\right)=\boldsymbol{\Phi}_{i}, i=1, \cdots, M$ and the FVs by $\mathbf{x}_{s_{j}}, j=1, \cdots, L$, note the corresponding images of FVs in $\mathbf{F}$ by $\boldsymbol{\Phi}\left(\mathbf{x}_{s_{j}}\right), j=1, \cdots, L$, where $L \leq M$. Then for the set of FVs $\mathbf{S}=\left\{\mathbf{x}_{s_{1}}, \mathbf{x}_{s_{2}}, \cdots, \mathbf{x}_{s_{L}}\right\}$, we can estimate the mapping of any sample as a linear combination of the images of $\mathbf{S}$ in $\mathbf{F}$. That is, the estimation $\hat{\boldsymbol{\Phi}}_{i}$ for $\boldsymbol{\Phi}_{i}$ can be formulated as a dot product:

$$
\hat{\boldsymbol{\Phi}}_{i}=\boldsymbol{\Phi}_{S} \boldsymbol{\alpha}_{i}
$$

In which $\boldsymbol{\Phi}_{S}=\left\{\boldsymbol{\Phi}\left(\mathbf{x}_{s_{1}}\right), \boldsymbol{\Phi}\left(\mathbf{x}_{s_{2}}\right), \cdots, \boldsymbol{\Phi}\left(\mathbf{x}_{s_{L}}\right)\right\}$ is a matrix composed with the images of FVs in $\mathbf{F} ; \boldsymbol{\alpha}_{i}=\left\{\alpha_{i 1}, \cdots, \alpha_{i L}\right\}$ is the corresponding coefficient vector to $\boldsymbol{\Phi}_{i}$. 
As mentioned above, our aim is to find a suitable set $\boldsymbol{\Phi}_{S}$ so that all the images of samples in $\mathbf{F}$ can be approximated as accurately as possible by Eq.(2). If the normalized Euclidean distance is used as evaluation criterion of difference between $\hat{\boldsymbol{\Phi}}_{i}$ and $\boldsymbol{\Phi}_{i}$, the problem of finding $\boldsymbol{\Phi}_{S}$ is finally transformed to find a set of samples $\mathbf{S}$ from the entire sample set which maximizes the fitness function $J_{s}[14]$ :

$$
J_{s}=\max _{\mathbf{S}} \frac{1}{M} \sum_{\mathrm{x}_{i} \in \mathbf{X}}\left(\frac{\mathbf{K}_{S i}^{t} \mathbf{K}_{S S}^{-1} \mathbf{K}_{S i}}{k_{i i}}\right)
$$

where $\mathbf{K}_{s s}$ is kernel matrix formed by FVs. $\mathbf{K}_{S i}=\left(k_{s_{p} i}\right)_{p=1, \cdots, L ; i=1, \cdots, M}$ is the kernel matrix calculated with $\mathbf{x}_{i}, i=1, \cdots, M$ and FVs. Eq. (3) is an optimization problem, whose solution could be obtained iteratively[14]. In each step, the support vector which is most orthogonal to the already-selected FVs set is chosen as a new feature vector. The iteration continues until an upper limitation value $J_{s}^{\max }, 0 \leq J_{s}^{\max } \leq 1$ of $J_{s}$ (or an upper limitation of ratio between number of FVs and SVs) is achieved. When $J_{s}^{\max }=1$, a complete basis of samples will be found; when $J_{s}^{\max }<1$, some unimportant FVs will be ignored and an approximated basis of samples will be found in $\mathbf{F}$. Interestingly, in previous experiments, we found ignoring unimportant FVs leads to the increase of the classification accuracy on the noised data by the obtained SVM[5]. The details about the implementation of FVS algorithm please refers to [14].

\subsection{Speedup of SVM Decision}

Next, let's turn our attention to the calculation of coefficient $\boldsymbol{\alpha}_{i}$ appeared in Eq. (2). By left production of $\boldsymbol{\Phi}_{S}^{t}$ to Eq.(2), one easily gets:

$$
\boldsymbol{\Phi}_{S}^{t} \hat{\boldsymbol{\Phi}}_{i}=\boldsymbol{\Phi}_{S}^{t} \boldsymbol{\Phi}_{S} \boldsymbol{\alpha}_{i} \Leftrightarrow \hat{\mathbf{K}}_{S i}=\mathbf{K}_{S S} \boldsymbol{\alpha}_{i}
$$

As mentioned above, $\hat{\boldsymbol{\Phi}}_{i}$ is a well approximation to $\boldsymbol{\Phi}_{i}$, so $\hat{\mathbf{K}}_{S i}$ can also be considered as a well approximation to $\mathbf{K}_{S i}$ and we denote $\hat{\mathbf{K}}_{S i}$ as $\mathbf{K}_{S i}$, and $\hat{\boldsymbol{\Phi}}_{i}$ as $\boldsymbol{\Phi}_{i}$ hereafter. Since $\mathbf{K}_{S S}$ is positively definite as a kernel matrix[1], $\mathbf{K}_{S S}^{-1}$, the inverse matrix of $\mathbf{K}_{S S}$ exists, so $\boldsymbol{\alpha}_{i}$ is obtained from Eq.(4) as:

$$
\boldsymbol{\alpha}_{i}=\mathbf{K}_{S S}^{-1} \mathbf{K}_{S i}
$$

then $\boldsymbol{\Phi}_{i}$ is formulated with $\boldsymbol{\Phi}_{S}$ as:

$$
\boldsymbol{\Phi}_{i}=\boldsymbol{\Phi}_{S} \mathbf{K}_{S S}^{-1} \mathbf{K}_{S i}
$$

Finally, let's try to express the decision function of SVM with FVs. The descision function of SVM is given as follows: 


$$
y=\operatorname{sgn}\left\{\sum_{j \in \mathbf{I}_{S V}} \beta_{j} y_{j}\left(\boldsymbol{\Phi}_{x}^{T} \boldsymbol{\Phi}_{j}\right)+b\right\}
$$

where $\operatorname{sgn}(x)=\left\{\begin{array}{l}+1, x \geq 0 \\ -1, x<0\end{array}\right.$ is the signal function, $\mathbf{I}_{S V}$ is the index set of SVs, $\beta_{j}$ is the corresponding Lagrange multiplier of SVs $\mathbf{x}_{j}, y_{j}$ is the class labels of $\mathbf{x}_{j}, b$ is the corresponding bias, and $\boldsymbol{\Phi}_{x}=\boldsymbol{\Phi}(\mathbf{x})$ is the image of the test sample $\mathbf{x}$ in $\mathbf{F}$. Substituting Eq. (6) into Eq. (7), one get:

$$
\begin{aligned}
y & =\operatorname{sgn}\left\{\sum_{j \in \mathbf{I}_{S V}} \beta_{j} y_{j}\left(\boldsymbol{\Phi}_{x}^{T} \mathbf{\Phi}_{S} \mathbf{K}_{S S}^{-1} \mathbf{K}_{S j}\right)+b\right\} \\
& =\operatorname{sgn}\left\{\sum_{j \in \mathbf{I}_{S V}} \beta_{j} y_{j}\left(\mathbf{K}_{S x}^{t} \mathbf{K}_{S S}^{-1} \mathbf{K}_{S j}\right)+b\right\} \\
& =\operatorname{sgn}\left\{\sum_{j \in \mathbf{I}_{S V}} \beta_{j} y_{j} \mathbf{K}_{S j}^{t} \mathbf{K}_{S S}^{-1} \mathbf{K}_{S x}+b\right\}
\end{aligned}
$$

After applying FVS method to the SVs set, both the SVs set and the FVs set $\boldsymbol{\Phi}_{S}$ are available, so $\mathbf{K}_{S j}^{t}$ and $\mathbf{K}_{S S}^{-1}$ can be calculated prior to the test. Let's denote $\mathbf{A}=\sum_{j \in \mathbf{I}_{S V}} \beta_{j} y_{j} \mathbf{K}_{S j}^{t} \mathbf{K}_{S S}^{-1}$, then $\mathbf{A}$ can also be calculated prior to the test and the Eq.(8) is simplified as:

$$
y=\operatorname{sgn}\left\{\mathbf{A} \mathbf{K}_{S x}+b\right\}
$$

It is obvious from Eq.(9) that to classify a new sample $\mathbf{x}$, the evaluation of kernel matrix of the new sample and the SVs is replaced by evaluation of kernel matrix of the sample $\mathbf{x}$ and FVs. As a subset of SVs set, the size of FVs set is always smaller than that of SVs, the test phase of the SVM can be speeded up by using Eq.(9).

\section{Experiments}

To clarify the speedup efficiency of the proposed method, the artificial and real world datasets from the IDA repository [15] are experimented. Every dataset was randomly separated into training and testing samples according to the predefined number ratio, each such a splits form a realization, and every dataset contains several realizations. The experiments are carried out on each realization and the averaged results are reported in this section. Experiments are completed on L2SVM with the commonly used RBF kernel, as more SVs are required by this type of SVM than the regular SVM (L1SVM) in the most situations [9], the reduction of SVs for L2SVM should be more necessary. The penalty coefficient $C$ and the width $\sigma$ of RBF function were chosen for the first 10 realizations of each dataset with "SVR+UD" method 
introduced in [16], and then the averaged $C$ and $\sigma$ over these 10 times choices are applied to each realization.

The method presented in this paper is very similar to that proposed by Downs T., Gates K.E. and Masters A.[12], the only difference is that in their method, a complete basis of SVs in feature space $\mathbf{F}$ is found and used to speed up the test phase of SVM while an approximated basis is used in present method. To clarify the difference of speedup performance between using complete and approximated basis, we first test the method of [12] with four datasets (Thyroid, Banana, Heart, Breast Cancer); the results are given in Table 1 . The reduction rate of SVs corresponding to bases is defined as:

$$
\text { Red. }=\frac{\# S V s-\# \text { Bases }}{\# S V s} \times 100
$$

Table 1. Optimized parameters ( $C, \sigma$ ), the averaged test error rate ( $R e$ ), the averaged number of SVs (\#SVs), the averaged number of bases (\#bases) and the number reduction rate of SVs respect to bases ( Red.) for four datasets

\begin{tabular}{ccccccc}
\hline \multirow{2}{*}{ Dataset } & \multicolumn{2}{c}{ Parameters } & \multirow{2}{*}{ Re / \% } & \#SVs & \#Bases & Red. / \% \\
\cline { 2 - 5 } & $C$ & $\sigma$ & & & & \\
\hline Thyroid & 0.89 & 1.14 & $3.47 \pm 1.87$ & 91.2 & 91.2 & 0 \\
Banana & 0.35 & 0.46 & $10.42 \pm 0.44$ & 339.59 & 302.73 & 10.85 \\
Heart & 0.42 & 4.39 & $16.00 \pm 3.18$ & 156.54 & 156.54 & 0 \\
BreastCancer & 7.6 & 2.1 & $22.92 \pm 4.48$ & 170.75 & 166.79 & 2.32 \\
\hline
\end{tabular}

It is shown by table 1 that in most cases, the size of the complete basis of SVs in $\mathbf{F}$ is very close to that of SVs set, so the test phase of SVM just can be speeded up very slightly. This suggests that in present method, we should use $J_{s}^{\max }<1$ (or set the upper limitation of the ratio between \#FVs and \#SVs smaller than 1) to find an incomplete basis so that the decision of SVM can be speeded up. In the next experiment, we tried various $J_{s}^{\max }$ on the above four datasets to find out its influence on the speedup efficiency and the classification performance; the results are listed in Table 2, where "\#FVs" is the averaged number of FVs over all realizations, "Re Incr." is the increase of the test error rate with respect to Table 1. The results show that the presented method can reduce the number of SVs obviously (about $40 \%$ $\sim 80 \%$ for different dataset) with only a minor loss in classification performance when $J_{s}^{\max }=0.98$; if the loss on classification is acceptable, more $20 \%$ reduction of SVs can be achieved by setting $J_{s}^{\max }=0.90$ for most datasets. In addition, Appendix A lists the best speedup ability of the proposed method on all 12 datasets of IDA on the premise of no obvious increase on the test error rate (the result of dataset "Titanic" is not presented here since the optimized parameters for this dataset can not be obtained by method of [16]). 
Table 2. Experimental results on various $J_{s}^{\max }$

\begin{tabular}{|c|c|c|c|c|c|}
\hline Dataset & $J_{s}^{\max }$ & $\operatorname{Re} 1 \%$ & Re Incr. $1 \%$ & \#FVs & Red. $1 \%$ \\
\hline \multirow{5}{*}{ Thyroid } & 0.98 & $3.49 \pm 1.83$ & 0.02 & 53.27 & 41.59 \\
\hline & 0.96 & $3.49 \pm 1.83$ & 0.02 & 53.27 & 41.59 \\
\hline & 0.94 & $3.52 \pm 1.70$ & 0.05 & 43.19 & 52.64 \\
\hline & 0.92 & $3.67 \pm 1.87$ & 0.20 & 40.54 & 55.55 \\
\hline & 0.90 & $3.88 \pm 1.90$ & 0.41 & 38.07 & 58.26 \\
\hline \multirow{5}{*}{ Banana } & 0.98 & $10.41 \pm 0.44$ & -0.01 & 63.18 & 81.40 \\
\hline & 0.96 & $10.46 \pm 0.46$ & 0.04 & 53.85 & 84.14 \\
\hline & 0.94 & $10.52 \pm 0.46$ & 0.10 & 48.46 & 85.73 \\
\hline & 0.92 & $10.54 \pm 0.49$ & 0.12 & 44.50 & 86.90 \\
\hline & 0.90 & $10.61 \pm 0.49$ & 0.19 & 41.29 & 87.84 \\
\hline \multirow{5}{*}{ Heart } & 0.98 & $16.03 \pm 3.19$ & 0.03 & 80.23 & 48.75 \\
\hline & 0.96 & $15.97 \pm 3.14$ & -0.03 & 61.27 & 60.86 \\
\hline & 0.94 & $15.81 \pm 3.09$ & -0.19 & 50.09 & 68.00 \\
\hline & 0.92 & $16.08 \pm 3.15$ & 0.08 & 42.18 & 73.05 \\
\hline & 0.90 & $16.23 \pm 3.08$ & 0.23 & 36.31 & 76.80 \\
\hline \multirow{5}{*}{$\begin{array}{l}\text { Breast } \\
\text { Cancer }\end{array}$} & 0.98 & $24.06 \pm 4.00$ & 1.14 & 103.12 & 39.61 \\
\hline & 0.96 & $24.19 \pm 3.93$ & 1.27 & 88.26 & 48.31 \\
\hline & 0.94 & $24.99 \pm 4.27$ & 2.07 & 78.71 & 53.90 \\
\hline & 0.92 & $25.45 \pm 4.24$ & 2.53 & 71.68 & 58.02 \\
\hline & 0.90 & $25.38 \pm 4.19$ & 2.46 & 65.93 & 61.39 \\
\hline
\end{tabular}

\section{Discussions}

A method which uses FVS method to reduce the number of SVs therefore the computaional complexity of SVM was described in this paper. Compared to the other post-processing speedup methods such as RS method $[2,10]$, our method has several advantages. Firstly, the founded FVs more "meaningful", they are a best approximated basis of SVs set in feature spaces. Secondly, since the FVS method try to find a minimum subset of SVs set to approximate each SVs, theoretically, the proposed method should reduce the complexity of SVM decision furthest among all methods based on the same idea (such as the method proposed in[12]). The third advantage of the proposed method is the potential denoise ability. We have proved in [5] that by ignore the "unimportant" FVs through setting $J_{s}^{\max }<1$, the classification accuracy on noisy data can be improved. The denosie ability of FVS method is proved again in the present experiments, for Heart and Banana dataset, we can find in table 2 that for some given $J_{s}^{\max }$, the classification error rate is also decreased.

Since the proposed method is a post-processing method and only operates on the SVs set, it is applicable for other type of kernels such as polynomial and sigmoid, 
and also applicable for other type of SVMs such as L1SVM, LS-SVM and so on; and also, it is applicable for both support vector classification and support vector regression. For the further speedup and better performance on claiification or regression, the optimized choice method for $J_{s}^{\max }$, e.g. goden search method, and retraining SVM with the obtained FVs[13], can be applied together with the present method.

Though the proposed methods possess above advantages, the FVS method is still a little expensive in calculation. In future, we plan to improve the present algorithm to make the method more practical in dealing with the large-scale classification problems. Another interesting phenomenon presented by the experiment results is that the dependency of the speedup ability of the present method on the dataset. For instance, the method can reduce number of FVs to 1 for dataset "Flare-solar" without any loss on the generalization ability of SVM while can only reduce $20.02 \%$ SVs for dataset "Image" with $0.25 \%$ increase on the test error rate. However, the reason for this phenomenon is still under investigation.

\section{Conclusions}

A new speedup method for SVM decision have been proposed in present paper. The method uses a subset which was chosen from SVs set by FVS algorithm to approximate the decision function of SVM. The trade-off between the speedup ability of the method and the final generaliation abiltiy of SVM can be easily controled by one parameter. As a post-processing method, the proposed method is applicalbe to any kind of SVM with various kernels. Experiments on IDA benchamrk datasets show that the number of SVs can be reduced $20 \%$ up to $99 \%$ with very slight loss in accuracy, and for some datasets, the reduction on SVs can lead to better classification accuracy.

Acknowledgements. The research is supported by the National Natural Search Foundation of China under Grant Number: 50575179.

\section{References}

1. Vapnik, V.N.: The Natural of Statistical Learning Theory. Springer-Verlag, Berlin Heidelberg New York (1995)

2. Burges, C. J. C.: Simplified support vector decision rules. Proc. 13th International Conference on Machine Learning San Mateo, CA. (1996) 71-77

3. Burges, C. J. C.: A tutorial on support vector machines for pattern recognition. Data Miningand Knowledge Discovery. 2 (1998) 121-167

4. Burges, C. J. C., Schoelkopf, B.: Improving the accuracy and speed of support vector learning machines. In: Mozer, M., Jordan, M. and Petsche, T. (eds.): Advances in neural information processing systems. Cambridge, MA: MIT Press. 9 (1997) 375-381

5. Zhu, Y. S., Zhang, Y. Y.: A new type SVM-Projected SVM. Science in China G: Physics, Mechanics \& Astronomy Supp. 47 (2004) 21-28

6. Osuna, E., Girosi, F.: Reducing the run-time complexity of Support Vector Machines. Proc.14th International Conference on Pattern Recognition. Btisbane, Austrilia (1998)

7. Tipping, M.E.: Sparse Bayesian Learning and the Relevance Vector Machine. Journal of Machine Learning Research. 1 (2001) 211-244 
8. Lee, Y.-J., Mangasarian, O. L.: RSVM: reduced support vector machines. in Proc. 1st SIAM Int. Conf. Data Mining.Chicago (2001)

9. Lin, K.-M.: A Study on Reduced Support Vector Machines. IEEE Trans. Neural Networks. 14 (2003) 1449-1459

10. Schoelkopf, B., Mika, S., Burges, C.J.C., Knirsch, P., Muller, K., Ratsch, G., Smola, A.J.: Input space versus feature space in kernel-based methods. IEEE Trans. Neural Networks. 10 (1999) 1000-1017.

11. Nguyen, D.D., Ho, T.B.: An Efficient Method for Simplifying Support Vector Machines. The 22nd International Conference on Machine Learning (ICML2005). Bonn, Germany (2005)

12. Downs, T., Gates, K.E., Masters, A.: Extract simplification of support vector solutions.Journal of Machine Learning Research. 2 (2001) 293-297

13. Suykens, J.A.K., Lukas, L., Vandewalle, J.: Sparese approximation using least squares support vector machines. In: Proc. of the IEEE International Symposium on Circuits and Systems. Geneva, Switzerland. (2000) II757-II760

14. Baudat, G., Anouar, F.: Feature Vector selection and projection using kernels. Neurocomputing. 55 (2003) 21-38

15. The datasets are from website: http://ida.first.gmd.de/ raetsch/data/benchmarks.htm

16. Zhu, Y.S., Li, C.H., Zhang Y.Y.: A practical parameters selection method for SVM. Lecture Notes in Computer Science (ISNN2004). 3173 (2004) 518-523

\section{Appendix A.}

The experimental results on IDA datasets, where column "L2SVM" gives the averaged test error rate of L2SVM without speedup; column "L2SVM+FVs" gives the averaged test error rate of L2SVM speeded up with the proposed method. "\#SVs" is the averaged number of SVs and "\#FVs" is the averaged number of FVs, "Red." is the number reduction rate of $\mathrm{FVs}$ respect to $\mathrm{SVs}$.

\begin{tabular}{cccccc}
\hline \multirow{2}{*}{ Dataset } & \multicolumn{2}{c}{ Test Error Rate (\%) } & \multicolumn{3}{c}{ Number of SVs (FVs) } \\
& L2SVM & L2SVM+FVS & \#SVs & \#FVs & Red. $(\%)$ \\
\hline Banana & $10.42 \pm 0.44$ & $10.61 \pm 0.49$ & 339.59 & 41.29 & 87.84 \\
Breast Cancer & $22.92 \pm 4.48$ & $24.06 \pm 4.00$ & 170.75 & 103.12 & 39.61 \\
Diabetis & $23.56 \pm 1.84$ & $23.75 \pm 1.84$ & 407.79 & 40.88 & 89.98 \\
Flare-solar & $34.75 \pm 2.74$ & $34.69 \pm 2.82$ & 109.23 & 1.00 & 99.08 \\
German & $23.73 \pm 2.21$ & $24.14 \pm 2.19$ & 599.95 & 150.11 & 74.98 \\
Heart & $16.00 \pm 3.18$ & $16.23 \pm 3.08$ & 156.54 & 36.31 & 76.80 \\
Image & $2.98 \pm 0.63$ & $3.23 \pm 0.67$ & 358.60 & 286.80 & 20.02 \\
Ringnorm & $2.03 \pm 0.27$ & $1.91 \pm 0.27$ & 141.72 & 58.91 & 58.43 \\
Splice & $11.58 \pm 0.72$ & $12.39 \pm 0.74$ & 553.70 & 387.7 & 29.98 \\
Thyroid & $3.47 \pm 1.87$ & $3.88 \pm 1.90$ & 91.2 & 38.07 & 58.26 \\
Twonorm & $2.52 \pm 0.15$ & $2.99 \pm 0.63$ & 113.32 & 20.33 & 82.06 \\
Waveform & $10.05 \pm 0.42$ & $10.99 \pm 0.74$ & 190.76 & 26.20 & 86.27 \\
\hline
\end{tabular}

